PROCEEDINGS OF THE

AMERICAN MATHEMATICAL SOCIETY

Volume 129, Number 2, Pages 593-599

S 0002-9939(00)05557-X

Article electronically published on July 27, 2000

\title{
DENSE SUBSETS OF MAXIMALLY ALMOST PERIODIC GROUPS
}

\author{
W. W. COMFORT AND SALVADOR GARCÍA-FERREIRA \\ (Communicated by Alan Dow)
}

\begin{abstract}
A (discrete) group $G$ is said to be maximally almost periodic if the points of $G$ are distinguished by homomorphisms into compact Hausdorff groups. A Hausdorff topology $\mathcal{T}$ on a group $G$ is totally bounded if whenever $\emptyset \neq U \in \mathcal{T}$ there is $F \in[G]^{<\omega}$ such that $G=U F$. For purposes of this abstract, a family $\mathcal{D} \subseteq \mathcal{P}(G)$ with $(G, \mathcal{T})$ a totally bounded topological group is a strongly extraresolvable family if (a) $|\mathcal{D}|>|G|$, (b) each $D \in \mathcal{D}$ is dense in $G$, and (c) distinct $D, E \in \mathcal{D}$ satisfy $|D \cap E|<d(G)$; a totally bounded topological group with such a family is a strongly extraresolvable topological group.
\end{abstract}

We give two theorems, the second generalizing the first.

Theorem 1. Every infinite totally bounded group contains a dense strongly extraresolvable subgroup.

Corollary. In its largest totally bounded group topology, every infinite Abelian group is strongly extraresolvable.

Theorem 2. Let $G$ be maximally almost periodic. Then there are a subgroup $H$ of $G$ and a family $\mathcal{D} \subseteq \mathcal{P}(H)$ such that

(i) $H$ is dense in every totally bounded group topology on $G$;

(ii) the family $\mathcal{D}$ is a strongly extraresolvable family for every totally bounded group topology $\mathcal{T}$ on $H$ such that $d(H, \mathcal{T})=|H|$; and

(iii) $H$ admits a totally bounded group topology $\mathcal{T}$ as in (ii).

Remark. In certain cases, for example when $G$ is Abelian, one must in Theorem 2 choose $H=G$. In certain other cases, for example when the largest totally bounded group topology on $G$ is compact, the choice $H=G$ is impossible.

\section{INTRODUCTION AND HISTORICAL REMARKS}

In the general topological study of topological groups there is an extensive literature addressing questions of the following form: Given properties $\mathbb{P}$ and $\mathbb{Q}$, does every topological group with $\mathbb{P}$ contain a (dense) subgroup with $\mathbb{Q}$ ? This paper participates in that tradition, with $\mathbb{P}$ the class of totally bounded groups and with

Received by the editors May 10, 1998 and, in revised form, April 23, 1999.

1991 Mathematics Subject Classification. Primary 22A05, 54A05, 54H11.

This work was written during the visit of the second-listed author to the Department of Mathematics of Wesleyan University, during the period September, 1997-March, 1998.

The second author acknowledges with thanks the generous hospitality and support received from the Department of Mathematics of Wesleyan University. 
$\mathbb{Q}$ the class of strongly extraresolvable groups. We turn to the necessary definitions and relevant background.

Embellishing and improving earlier theorems of Hewitt [8], Ceder [2] showed that every metrizable space without isolated points, and every locally compact Hausdorff space without isolated points, is maximally resolvable in the following sense: Each such space $X=(X, \mathcal{T})$ admits a family of $\Delta(X)$-many pairwise disjoint dense subsets; here $\Delta(X)$, the so-called dispersion character of $X$, is the cardinal number

$$
\Delta(X)=\min \{|U|: \emptyset \neq U \in \mathcal{T}\} .
$$

Evidently Ceder's word "maximal" is fully justified and well-deserved: No pairwise disjoint family $\mathcal{D}$ of dense subsets of $X$ can satisfy $|\mathcal{D}|>\Delta(X)$. Subsequently however Malykhin [10] suggested the usefulness of considering a family $\mathcal{D}$ of dense subsets of a space $X$, with $|\mathcal{D}|>\Delta(X)$, whose pairwise intersections, though not necessarily empty, are "small" in some appropriate sense. Malykhin focussed on the property nowhere dense; other possibilities, topological or measure-theoretic in nature, will occur to the reader.

Specifically, Malykhin [10] calls a space extraresolvable if there is a family $\mathcal{D} \subseteq$ $\mathcal{P}(X)$ of dense subsets of $X$ such that $|\mathcal{D}|>\Delta(X)$ and every pair of distinct elements of $\mathcal{D}$ has nowhere dense intersection. [We were informed in an e-mail message in May, 1998 from Professor Malykhin that in [11, [12] he has refined and advanced the results of [10]. He has found for example in every countable totally bounded group a "weak sequence of finite sets converging" to each point of the group, thus establishing simultaneously the extraresolvability of each totally bounded group topology on each countable group.] We here propose a narrower, more restrictive property; it not only demands that the space is extraresolvable in the sense of Malykhin, but it also explains why it is extraresolvable.

Definition. A space $X$ is strongly extraresolvable if there is a family $\mathcal{D}$ of dense subsets of $X$ such that $|\mathcal{D}|>\Delta(X)$ and every pair $D, E$ of distinct elements of $\mathcal{D}$ satisfy $|D \cap E|<n w d(X)$; here $n w d(X)$, the nowhere density number of $X$, is the cardinal number defined by

$$
\operatorname{nwd}(X)=\min \{|A|: A \subseteq X, A \text { is not nowhere dense in } X\} .
$$

Such a family $\mathcal{D} \subseteq \mathcal{P}(X)$ is called a strongly extraresolvable family on $X$.

Throughout this paper, for $X$ a set and $\kappa$ a cardinal number, we write $[X]^{\kappa}=$ $\{A \subseteq X:|A|=\kappa\}$. The notations $[X]^{\leq \kappa}$ and $[X]^{<\kappa}$ are defined analogously.

Following von Neumann [14], we say that a (discrete) group $G$ is maximally almost periodic if the points of $G$ are distinguished by continuous homomorphisms into compact Hausdorff groups. (Abelian groups have that property, but certain other groups do not. For example, von Neumann and Wigner [15] showed that every homomorphism from the matrix group $S L(2, \mathbb{Q})$ to a compact group is trivial. See also von Neumann [14](§18) for a similar conclusion concerning the groups $S L(n, \mathbb{R})$ for $2 \leq n<\omega$.) Evidently a maximally almost periodic group $G$ embeds algebraically into a compact Hausdorff group. Each topology $\mathcal{T}$ which $G$ inherits from a compact group is totally bounded in the sense that whenever $\emptyset \neq U \in \mathcal{T}$ there is $F \in[G]^{<\omega}$ such that $G=U F$. Presenting theorems of Malykhin based on a novel construction of Protasov, those authors show [13] that every totally bounded topological group $(G, \mathcal{T})$ is maximally resolvable; indeed there is a pairwise disjoint family $\mathcal{D} \subseteq[\mathcal{P}(G)]^{\Delta(G)}$ such that each $D \in \mathcal{D}$ is dense simultaneously in each totally 
bounded group topology on $G$. (Though the point is not made in [13], this stronger statement follows from the weaker one: Every maximally almost periodic group admits a largest totally bounded group topology $t(G)$ - specifically, the topology induced on $G$ by all homomorphisms into compact groups - and it is clear that any $t(G)$-dense subset of $G$ is dense in every totally bounded group topology on $G$.)

In the present paper we show (Theorem 2.9) that every maximally almost periodic group $G$ contains a subgroup $H$ with a family $\mathcal{D} \subseteq \mathcal{P}(H)$ such that $H$ is dense in every totally bounded group topology on $G, d(H, t(H))=|H|$, and $H$ admits a family of subsets $\mathcal{D}$ which is strongly extraresolvable for every totally bounded group topology $\mathcal{T}$ on $H$ satisfying $d(H, \mathcal{T})=|H|$.

1.1. Remark. Results of this and of our related manuscript 3 were announced at the URLs www .unipissing.ca/topology/i/a/a/c/53.htm and www. unipissing . ca/topology/i/a/a/c/55.htm of the Topology Atlas website.

\section{Dense SUBSETS WITH NOWHERE DENSE INTERSECTION}

Our first lemma strengthens a familiar statement (see 7](12.8), for example).

2.1. Lemma. Let $\alpha \geq \omega$, let $\left\{A_{\xi}: \xi<\alpha\right\}$ be a (faithfully indexed) family of pairwise disjoint sets with each $\left|A_{\xi}\right|=\alpha$, and let $X=\bigcup_{\xi<\alpha} A_{\xi}$. Then there is a family $\mathcal{E} \subseteq \mathcal{P}(X)$ such that

(i) $|\mathcal{E}|=\alpha^{+}$;

(ii) $\left|E \cap A_{\xi}\right|=1$ whenever $E \in \mathcal{E}, \xi<\alpha$; and

(iii) $|D \cap E|<\alpha$ whenever $D \in \mathcal{E}, E \in \mathcal{E}$, and $D \neq E$.

Proof. Clearly there is a pairwise disjoint family $\mathcal{D} \subseteq \mathcal{P}(X)$ satisfying (the analogues of) (ii) and (iii) such that $|\mathcal{D}|=\alpha$. Zorn's Lemma then gives a family $\mathcal{E}$ of sets maximal with respect to conditions $\left(\mathrm{i}^{\prime}\right)$, (ii) and (iii) where $\left(\mathrm{i}^{\prime}\right)$ is the condition

$\left(\mathrm{i}^{\prime}\right) \mathcal{D} \subseteq \mathcal{E} \subseteq \mathcal{P}(X)$.

To show that $|\mathcal{E}| \geq \alpha^{+}$, suppose instead that $\mathcal{E}$ may be indexed by $\alpha$, say $\mathcal{E}=$ $\left\{E_{\eta}: \eta<\alpha\right\}$. For $\xi<\alpha$ we have $\left|A_{\xi}\right|=\alpha$ and $\left|A_{\xi} \cap\left(\bigcup_{\eta \leq \xi} E_{\eta}\right)\right| \leq|\xi|<\alpha$, so there is $x_{\xi} \in A_{\xi} \backslash \bigcup_{\eta \leq \xi} E_{\eta}$. The set $E:=\left\{x_{\xi}: \xi<\alpha\right\}$ evidently satisfies (ii), and $\left|E \cap E_{\eta}\right| \leq|\eta|<\alpha$ for each $\eta<\alpha$ (whence $E \notin \mathcal{E}$ ), contrary to the maximality of $\mathcal{E}$.

In what follows for a group $G$ we write $\mathcal{F}=\mathcal{F}(G)=\left\{F \in[G]^{<\omega}: F=F^{-1}\right\}$. The next lemma has been noted in [13].

2.2. Lemma. Let $G=(G, \mathcal{T})$ be a totally bounded topological group and let a $\in$ $G^{\mathcal{F}}$. Then $D:=\bigcup_{F \in \mathcal{F}} a(F) \cdot F$ is dense in $(G, \mathcal{T})$.

Proof. Given $\emptyset \neq U \in \mathcal{T}$ there is $F \in \mathcal{F}$ such that $U \cdot F=G$. Then with $a(F) \in u F$ $(u \in U)$ we have $u \in\left(a(F) \cdot F^{-1}\right) \cap U=(a(F) \cdot F) \cap U \subseteq D \cap U$, as required.

Next comes the combinatorial lemma upon which the proof of Theorem 2.4 chiefly depends.

2.3. Lemma. For every group $G$ with $|G|=\alpha \geq \omega$ and for every indexing $\left\{F_{\xi}: \xi<\alpha\right\}$ of $\mathcal{F}(G)$ there is a family $\left\{A_{\xi}: \xi<\alpha\right\} \subseteq \mathcal{P}(G)$ such that

(i) each $\xi<\alpha$ satisfies $\left|A_{\xi}\right|=\alpha$;

(ii) distinct $\xi, \xi^{\prime}<\alpha$ satisfy $A_{\xi} \cap A_{\xi^{\prime}}=\emptyset$;

(iii) distinct $\xi$, $\xi^{\prime}<\alpha$ satisfy $A_{\xi} \cdot F_{\xi} \cap A_{\xi^{\prime}} \cdot F_{\xi^{\prime}}=\emptyset$; and

(iv) distinct $a, a^{\prime} \in A_{\xi}$ satisfy $\left(a \cdot F_{\xi}\right) \cap\left(a^{\prime} \cdot F_{\xi}\right)=\emptyset$. 
Proof. Write $L:=\{\langle\eta, \xi\rangle: \xi \leq \eta<\alpha\}$ and give $L$ the usual lexicographic ordering: $\left\langle\eta^{\prime}, \xi^{\prime}\right\rangle<\langle\eta, \xi\rangle$ if either (a) $\eta^{\prime}<\eta$, or (b) $\eta^{\prime}=\eta$ and $\xi^{\prime}<\xi$. By recursion, for $\langle\eta, \xi\rangle \in L$ we will define $S(\eta, \xi) \subseteq G$ and $a_{\eta, \xi} \in G$.

Let $S(0,0)=\emptyset$ and let $a_{0,0}$ be arbitrary in $G$.

Now let $\langle\eta, \xi\rangle \in L$, suppose that $S\left(\eta^{\prime}, \xi^{\prime}\right)$ and $a_{\eta^{\prime}, \xi^{\prime}}$ have been defined for all $\left\langle\eta^{\prime}, \xi^{\prime}\right\rangle<\langle\eta, \xi\rangle$, and define

$$
S(\eta, \xi)=\left\{a_{\eta^{\prime}, \xi^{\prime}}:\left\langle\eta^{\prime}, \xi^{\prime}\right\rangle<\langle\eta, \xi\rangle\right\} \cup\left(\bigcup\left\{a_{\eta^{\prime}, \xi^{\prime}} \cdot F_{\xi^{\prime}} \cdot F_{\xi}:\left\langle\eta^{\prime}, \xi^{\prime}\right\rangle<\langle\eta, \xi\rangle\right\}\right) .
$$

When $\eta<\omega$, we have $|S(\eta, \xi)|<\omega \leq \alpha$ and when $\omega \leq \eta<\alpha$, we have $|S(\eta, \xi)| \leq$ $|\eta|^{2}+|\eta|^{2} \cdot \omega<\alpha$; thus there is $a_{\eta, \xi} \in G \backslash S(\eta, \xi)$.

The definitions of $S(\eta, \xi)$ and $a_{\eta, \xi}$ for $\langle\eta, \xi\rangle \in L$ are complete. For $\xi<\alpha$ we define $A_{\xi}=\left\{a_{\eta, \xi}: \xi \leq \eta<\alpha\right\}$ and we verify (i), (ii), (iii) and (iv).

(i) For $\eta^{\prime}<\eta<\alpha$, from $\left\langle\eta^{\prime}, \xi\right\rangle<\langle\eta$, $\xi\rangle$ we have $a_{\eta^{\prime}, \xi} \in S(\eta, \xi)$ and hence $a_{\eta^{\prime}, \xi} \neq a_{\eta, \xi}$. Thus $\left|A_{\xi}\right|=\alpha$.

(ii) and (iii). Let $\xi \neq \xi^{\prime}$ and let $a_{\eta^{\prime}, \xi^{\prime}} \in A_{\xi^{\prime}}$ and $a_{\eta, \xi} \in A_{\xi}$. Then, assuming without loss of generality that $\left\langle\eta^{\prime}, \xi^{\prime}\right\rangle<\langle\eta, \xi\rangle$, we have $a_{\eta^{\prime}, \xi^{\prime}} \in S(\eta, \xi)$ and $a_{\eta^{\prime}, \xi^{\prime}}$. $F_{\xi^{\prime}} \cdot F_{\xi} \subseteq S(\eta, \xi)$. Then from $a_{\eta, \xi} \notin S(\eta, \xi)$ follows

$$
a_{\eta, \xi} \neq a_{\eta^{\prime}, \xi^{\prime}}
$$

and

$$
\left(a_{\eta, \xi} \cdot F_{\xi}\right) \cap\left(a_{\eta^{\prime}, \xi^{\prime}} \cdot F_{\xi^{\prime}}\right)=\left(a_{\eta, \xi} \cdot F_{\xi}^{-1}\right) \cap\left(a_{\eta^{\prime}, \xi^{\prime}} \cdot F_{\xi^{\prime}}\right)=\emptyset .
$$

(iv) Suppose without loss of generality that $a=a_{\eta, \xi}$ and $a^{\prime}=a_{\eta^{\prime}, \xi}$ with $\eta^{\prime}<\eta$. Then $\left\langle\eta^{\prime}, \xi\right\rangle<\langle\eta, \xi\rangle$, and from $a_{\eta^{\prime}, \xi} \cdot F_{\xi} \cdot F_{\xi} \subseteq S(\eta, \xi)$ and $a_{\eta, \xi} \notin S(\eta, \xi)$ follows

$$
\left(a \cdot F_{\xi}\right) \cap\left(a^{\prime} \cdot F_{\xi}\right)=\left(a_{\eta, \xi} \cdot F_{\xi}^{-1}\right) \cap\left(a_{\eta^{\prime}, \xi} \cdot F_{\xi}\right)=\emptyset .
$$

As usual for a space $X$ we write

$$
d(X)=\min \{|A|: A \text { is dense in } X\} .
$$

2.4. Theorem. Let $G=(G, \mathcal{T})$ be a totally bounded topological group such that $|G|=d(G)=\alpha \geq \omega$. Then $G$ is strongly extraresolvable.

Proof. From the fact that $G$ is covered by finitely many translates of each nonempty $U \in \mathcal{T}$ it is immediate that $\Delta(G)=|G|=\alpha$ and that $n w d(G)=d(G)=\alpha$.

Let $\left\{F_{\xi}: \xi<\alpha\right\}$ be an indexing of $\mathcal{F}(G)$, let $\left\{A_{\xi}: \xi<\alpha\right\} \subseteq[G]^{\alpha}$ be as given by Lemma 2.3, and (using Lemma 2.1) let $\mathcal{E}=\left\{E_{\eta}: \eta<\alpha^{+}\right\}$be a faithfully indexed subset of $[G]^{\alpha}$ such that $\left|E_{\eta} \cap A_{\xi}\right|=1$ for $\eta<\alpha^{+}, \xi<\alpha$, and $\left|E_{\eta} \cap E_{\eta^{\prime}}\right|<\alpha$ whenever $\eta<\eta^{\prime}<\alpha^{+}$. Abandoning earlier numeration, for notational convenience we now let $E_{\eta} \cap A_{\xi}=\left\{a_{\eta, \xi}\right\}$, and we set

$$
D_{\eta}=\bigcup\left\{a_{\eta, \xi} \cdot F_{\xi}: \xi<\alpha\right\} \text { for } \eta<\alpha^{+} .
$$

Since $D_{\eta}$ is dense in $G$ by Lemma 2.2 , it remains only to check that $\left|D_{\eta^{\prime}} \cap D_{\eta}\right|<\alpha$ whenever $\eta^{\prime}<\eta<\alpha$. We have

$$
D_{\eta^{\prime}} \cap D_{\eta}=\left[\bigcup_{\xi^{\prime}<\alpha} a_{\eta^{\prime}, \xi^{\prime}} \cdot F_{\xi^{\prime}}\right] \cap\left[\bigcup_{\xi<\alpha} a_{\eta, \xi} \cdot F_{\xi}\right]
$$


When $\xi \neq \xi^{\prime}$ we have $a_{\eta^{\prime}, \xi^{\prime}} \cdot F_{\xi^{\prime}} \cap a_{\eta, \xi} \cdot F_{\xi}=\emptyset$ by condition (iii) of Lemma 2.4 , so $(*)$ reduces to

$$
D_{\eta^{\prime}} \cap D_{\eta}=\bigcup_{\xi<\alpha}\left[\left(a_{\eta^{\prime}, \xi} \cdot F_{\xi}\right) \cap\left(a_{\eta, \xi} \cdot F_{\xi}\right)\right] .
$$

If $\xi<\alpha$ satisfies $\left(a_{\eta^{\prime}, \xi} \cdot F_{\xi}\right) \cap\left(a_{\eta, \xi} \cdot F_{\xi}\right) \neq \emptyset$, then from condition (iv) of Lemma 2.4 we have $a_{\eta^{\prime}, \xi}=a_{\eta, \xi} \in E_{\eta^{\prime}} \cap E_{\eta}$. Thus relation (**) expresses $D_{\eta^{\prime}} \cap D_{\eta}$ as the union of $\left|E_{\eta^{\prime}} \cap E_{\eta}\right|$-many finite sets, and from $\left|E_{\eta^{\prime}} \cap E_{\eta}\right|<\alpha$ we conclude $\left|D_{\eta^{\prime}} \cap D_{\eta}\right|<\alpha$, as required.

2.5. Theorem. Every infinite totally bounded topological group admits a dense strongly extraresolvable subgroup.

Proof. Given such a group $G$ let $H$ be a dense subgroup of $G$ such that $|H|=d(G)$. Then $|H|=d(H)$, so Theorem 2.4 applies to $H$.

2.6. Remark. From Theorem 2.5 it follows that every infinite totally bounded topological group with no proper dense subgroup is strongly extraresolvable. The interested reader might consult 44 for a (presumably incomplete) list of Abelian groups which satisfy that criterion. Long before the appearance of [4] it was known that every infinite Abelian $G$, in the topology induced by $\operatorname{Hom}(G, \mathbb{T})$, has the property that each subgroup is closed. (Equipped with this topology, the so-called Bohr topology, $G$ is often denoted $G^{\#}$; this notation and many early relevant results and questions are due to van Douwen [5.).) Because of the interest which the groups $G^{\#}$ have generated, we record this special instance of Theorem 2.5 explicitly.

2.7. Corollary. Let $G$ be an infinite Abelian group. Then $G^{\#}$ is strongly extraresolvable.

Finally in Theorem 2.9 we note that with minimal additional effort Theorem 2.5 can be sharpened and enhanced. (Theorem 2.9 is the result stated as Theorem 2 in the abstract.)

2.8. Remark. In contrast to the necessary relation $H=G$ with $G$ as in 2.6 and 2.7, the inclusion $H \subseteq G$ of Theorem 2.5 must be proper when $G$ is (infinite and) compact. Indeed it is noted in [6] that such a group $G$ cannot even be extraresolvable. To see this let $w(G)=\alpha$ and with $\mathcal{D}$ a family of dense subsets of $G$ choose for each $D \in \mathcal{D}$ a dense subset $E(D) \subseteq D$ such that $|E(D)| \leq \alpha$. Since $|[G] \leq \alpha|=\left(2^{\alpha}\right)^{\alpha}=2^{\alpha}=\Delta(G)$, the map $D \rightarrow E(D)$ from $\mathcal{D}$ into $\mathcal{P}(G)$ cannot be an injection if $|\mathcal{D}|>2^{\alpha}$.

2.9. Theorem. Let $G$ be an infinite maximally almost periodic group. Then there are a subgroup $H$ of $G$ and a family $\mathcal{D} \subseteq \mathcal{P}(H)$ such that

(i) $H$ is dense in every totally bounded group topology on $G$;

(ii) the family $\mathcal{D}$ is a strongly extraresolvable family for every totally bounded group topology $\mathcal{T}$ on $H$ such that $d(H, \mathcal{T})=|H|$; and

(iii) $H$ admits a totally bounded group topology $\mathcal{T}$ as in (ii).

Proof. Let $t(G)$ be the largest totally bounded group topology on $G$, let $H$ be a dense subgroup of $(G, t(G))$ such that $|H|=d(G, t(G))$, and let $t(H)$ be the largest totally bounded group topology on $H$. From $t(G) \mid H \subseteq t(H)$ follows

$$
|H|=d(H, t(G) \mid H) \leq d(H, t(H)) \leq|H|,
$$


so by Theorem 2.4 there is a strongly extraresolvable family $\mathcal{D}$ for $(H, t(H))$. For every totally bounded group topology $\mathcal{T}$ on $H$ every $D \in \mathcal{D}$ is $\mathcal{T}$-dense in $H$ (being $t(H)$-dense), so if in addition $d(H, \mathcal{T})=|H|$, then just as in the first line of the proof of Theorem 2.4 we have $\Delta(H, \mathcal{T})=\Delta(H, t(G) \mid H)=|H|$ and $n w d(H, \mathcal{T})=$ $n w d(H, t(G) \mid H)=|H|$; thus $\mathcal{D}$ is a strongly extraresolvable family for $(H, \mathcal{T})$.

2.10. Remarks. (a) It is interesting to notice, though logically inessential to our work, that in Theorem 2.9 the inclusion $t(G) \mid H \subseteq t(H)$ may be proper. For an example to this effect recall from van der Waerden 17] that every homomorphism from the compact metric group $G=S O(3, \mathbb{R})$ to a compact group is continuous; thus $t(G)$ is the usual compact topology of $G$. It is well known and geometrically clear that $G$ contains densely a copy of the free group $F(2)$; indeed according to Auerbach's theorem [1] as exposed by Hofmann and Morris [9](6.52), the set of points $(x, y) \in G \times G$ for which the subgroup of $G$ generated by $\{x, y\}$ is dense in $G$ and free on $\{x, y\}$ contains a dense set of the form $\bigcap_{n<\omega} U_{n}$ with each $U_{n}$ dense and open in $G \times G$. Now let $H$ be any copy of $F(2)$ dense in $G$. Remus [16] has shown that the group $(H, t(H))$ is not metrizable; indeed according to Remus [16] (2.18) there are $2^{\mathfrak{c}}$-many totally bounded group topologies $\mathcal{T}$ on $F(2)$ for which $w(F(2), \mathcal{T})=\mathfrak{c}$. It then follows that $w(H, t(H))=\mathfrak{c}$ and the inclusion $t(G) \mid H \subseteq t(H)$ is proper (since $w(H, t(G) \mid H)=\omega$ ).

The same example shows that not every maximally almost periodic group $G$ admits a totally bounded group topology $\mathcal{T}$ for which $d(G, \mathcal{T})=|G|$. Indeed with respect to the unique totally bounded group topology on $S O(3, \mathbb{R})$ we have $d(S O(3, \mathbb{R}))=\omega<\mathfrak{c}=|S O(3, \mathbb{R})|$.

(b) Our results concerning maximally almost periodic groups apply of course to groups which admit a compact Hausdorff group topology, but there are severe limitations to extending the argument to locally compact groups: Every such (nondiscrete) group admits a dense extraresolvable subset, but not all such groups admit a dense extraresolvable subgroup; and there are locally compact nondiscrete groups with no dense strongly extraresolvable subset. For these and related results, including examples of extraresolvable groups which are not strongly extraresolvable, the interested reader may consult our paper [3].

\section{ACKNOWLEDGEMENT}

We are grateful to Oscar Masaveu, Dieter Remus, and Lewis C. Robertson for instructive conversation and for helpful comments on a preliminary version of this manuscript.

\section{REFERENCES}

[1] H. Auerbach, Sur les groupes linéaires bornés, Studia Math. 5 (1934), 43-49.

[2] J. G. Ceder, On maximally resolvable spaces, Fund. Math. 55 (1964), 87-93. MR 29:582

[3] W. W. Comfort and Salvador García-Ferreira, Strongly extraresolvable groups and spaces, Proc. Mexico City Summer (1998) Topology Conference. Topology Proceedings 23. To appear.

[4] W. W. Comfort and Jan van Mill, Groups with only resolvable group topologies, Proc. Amer. Math. Soc. 120 (1993), 687-696. MR 94f:20107

[5] E. K. van Douwen, The maximal totally bounded group topology on $G$ and the biggest minimal $G$-space for Abelian groups $G$, Topology Appl. 34 (1990), 69-91.

[6] S. García-Ferreira, V. I. Malykhin and A. Tomita, Extraresolvable spaces. Topology and Its Applications. 1999. To Appear. 
[7] Leonard Gillman and Meyer Jerison, Rings of Continuous Functions. D. Van Nostrand Co., New York. 1960. MR 22:6994

[8] Edwin Hewitt, A problem of set-theoretic topology, Duke Math. J. 10 (1943), 309-333. MR 5:46e

[9] Karl Heinrich Hofmann and Sidney A. Morris, The Structure of Compact Groups; A Primer for Students, A Handbook for Experts. de Gruyter, Berlin. 1998. MR 99k:22001

[10] V. I. Malykhin, Irresolvability is not descriptively good. Manuscript submitted for publication. 1996.

[11] V. I. Malykhin, Something new about totally bounded groups, Mat. Zametki 65 (1999), 474476. [In Russian].

[12] V. I. Malykhin, To the combinatorial analysis of infinite groups, Manuscript in Preparation. 1998.

[13] V. I. Malykhin and I. V. Protasov, Maximal resolvability of bounded groups, Topology Appl. 73 (1996), 227-232. MR 97k:54030

[14] J. von Neumann, Almost periodic functions in a group I, Trans. Amer. Math. Soc. 36 (1934), 445-492.

[15] J. von Neumann and E. P. Wigner, Minimally almost periodic groups, Annals of Math. (Series 2) 41 (1940), 746-750. MR 2:127e

[16] Dieter Remus, Minimal and precompact group topologies on free groups, J. Pure and Applied Algebra 70 (1991), 147-157. MR 92h:22004

[17] B. L. van der Waerden, Stetigkeitssätze für halbeinfache Liesche Gruppen, Math. Zeitschrift 36 (1933), 780-786.

Department of Mathematics, Wesleyan University, Middletown, Connecticut 06459

E-mail address: wcomfort@wesleyan.edu

Instituto de Matemáticas, Ciudad Universitaria (UNAM), 04510 México D.F., México

E-mail address: garcia@servidor.unam.mx

E-mail address: sgarcia@zeus.ccu.umich.mx 